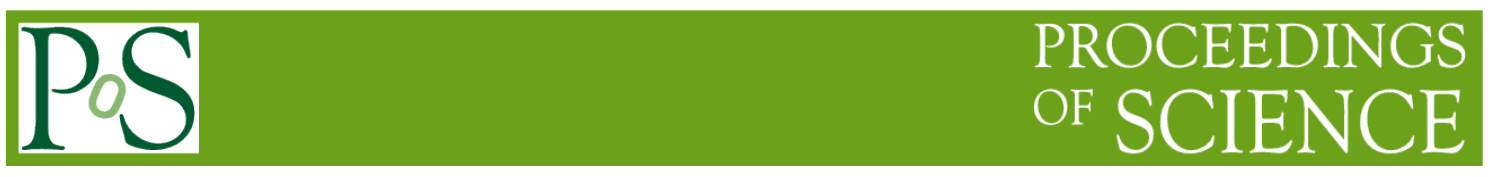

\title{
Apertif; the next stage
}

\section{Tom Oosterloo}

ASTRON

Oude Hoogeveensedijk 4, 7991 PD Dwingeloo, The Netherlands E-mail: oosterloo@astron.nl

\section{Joeri van Leeuwen}

ASTRON

Oude Hoogeveensedijk 4, 7991 PD Dwingeloo, The Netherlands

E-mail: leeuwen@astron.nl

\section{Wim van Cappellen}

ASTRON

Oude Hoogeveensedijk 4, 7991 PD Dwingeloo, The Netherlands

E-mail: cappellen@astron.nl

\section{Gert Kruithof}

ASTRON

Oude Hoogeveensedijk 4, 7991 PD Dwingeloo, The Netherlands

E-mail: kruithof@astron.nl

\section{Carole Jackson}

\section{ASTRON}

Oude Hoogeveensedijk 4, 7991 PD Dwingeloo, The Netherlands

E-mail: jackson@astron.nl

50 Years Westerbork Radio Observatory, A Continuing Journey to Discoveries and Innovations Richard Strom, Arnold van Ardenne, Steve Torchinsky (eds) 


\section{Apertif; the next stage}

\section{Chapter 15.1 Observing Perspectives with Apertif}

Tom Oosterloo*

$\mathrm{R}$ adio astronomy has made several fundamental contributions to science. Among these are the detection of the cosmic microwave background, the discovery of pulsars, revealing the existence of active galactic nuclei, and detailed spectroscopic studies of the kinematics of the gas in galaxies which played an important role in the discovery of dark matter. In order to maintain this momentum of discovery, the performance of radio telescopes has to be improved continuously. One of the main limitations of current radio telescopes is their relatively poor performance for doing deep, high spatial resolution surveys of large regions of the sky. In many other wave bands, very large data sets resulting from large surveys covering large fractions of the sky are now available. These data sets have a large impact because they enable statistical studies on a scale not possible before, while they also are, because of their scale, rich sources of rare (and thus interesting) types of objects. The fact that the data from these surveys are publicly available is an important additional factor contributing to their success. This has, to some extent, made astronomy more democratic. Instead of data being available to only those who have access to a telescope, everybody can just take the data from a public archive.

So far, radio astronomy has played a relatively limited role in this 'new' astronomy which is based on large public surveys. A number of important radio surveys are available (e.g., WENSS, NVSS, FIRST, HIPASS, Alfalfa) but they are, compared to the quality and depth of the large surveys in other wave bands, of limited sensitivity and/or spatial resolution. This will change, of course, with the advent of the Square Kilometre Array (SKA), but in the mean time significant improvements can already be realised. One of the main reasons radio surveys are lagging behind those in other branches of astronomy is that, given the sensitivity and field of view of current radio telescopes, it is very expensive in terms of amounts of observing time to survey large volumes of space with sufficiently high sensitivity and resolution. Hence, the available radio surveys are either relatively shallow or have very low resolution, or both. An obvious

* ASTRON, The Netherlands solution to this problem is to enlarge the field of view of a radio telescope so that large areas can be surveyed much faster. ASTRON has been leading such 
efforts with LOFAR at low frequencies and with Apertif in the 1-2 GHz band. Both instruments will produce very large datasets covering much of the sky and it is likely that these data sets will have a major impact on how radio astronomy is done, as well as on the role of radio data in modern survey astronomy. Given the large frequency range the combined data sets from LOFAR and Apertif will cover, there is, in fact, a very useful and unique synergy between both instruments. They will also play an important role in preparing radio astronomy for the era of the SKA. Below, we briefly describe the main science topics of Apertif in the context of the issues discussed above.

\section{Gas and galaxy evolution}

One of the main scientific applications of Apertif is to observe large volumes of space in order to make a full inventory of the detailed properties of the neutral hydrogen (HI) in galaxies and see what these can tell about the structure and evolution of galaxies. Detailed spatially resolved studies of the HI in galaxies has been one of the key science areas of the WSRT since the 1970s and these have greatly contributed to improving our understanding of galaxies. However, although these studies have been very successful, these HI surveys, because of the characteristics of the WSRT, have been limited to relatively small samples of galaxies in the nearby Universe (e.g. the WHISP and ATLAS ${ }^{2}$ surveys) and do not match the large surveys in other wavebands. A few large surveys of the HI content of galaxies have been done with other instruments (HIPASS with Parkes and Alfalfa with Arecibo), but these are based on observations with single-dish telescopes which do not spatially resolve the objects and hence give much less information on the internal distribution and kinematics of the $\mathrm{HI}$ in galaxies. They only provide the global HI properties, such as total HI mass and overall rotation velocity. Interesting science can be done with such data and many interesting results have been obtained, in particular concerning global scaling relations such as how the gas content varies with galaxy mass and other global galaxy properties. But these data are less suitable for studying which phenomena drive the existence of these scaling relations because one cannot see what is going on in the galaxies. The key improvement Apertif will bring is that it will provide a very large database of spatially resolved information on the $\mathrm{HI}$ in galaxies instead of on global properties only. Hence, not only can the scaling relations be investigated, but in addition also the mechanisms responsible for these scaling relations because the processes occurring in the galaxies can be imaged. This huge improvement will bring extragalactic HI studies to a different level and makes it possible to much better understand the scaling relations. Figure 1, taken from a project done with the old WSRT to prepare for Apertif, illustrates what a typical Apertif HI detection will look like and the kind of information Apertif will give on a very large number of galaxies.

With such information, the properties of the neutral hydrogen in galaxies as function of mass, type and environment can be studied in much better detail. For example, analysis of the Alfalfa survey has shown that larger galaxies

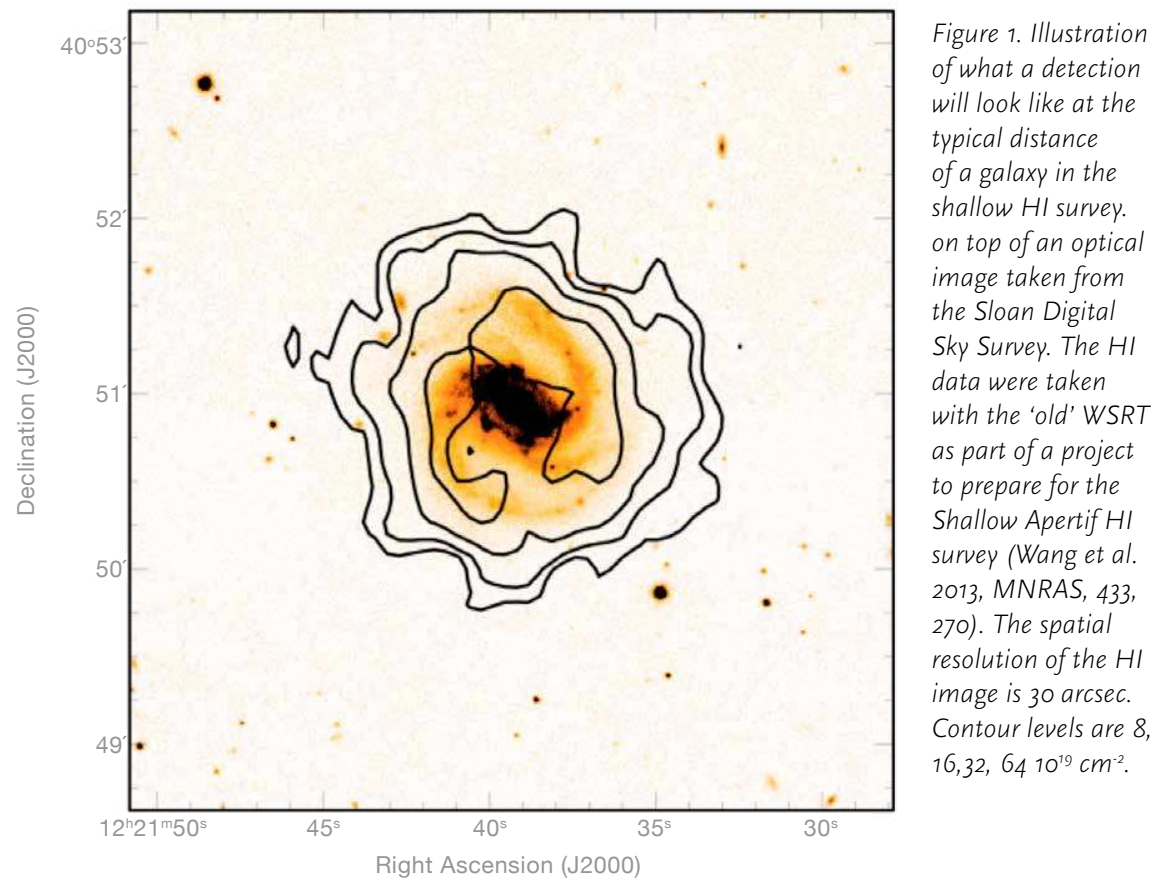

(i.e. galaxies with more stars) have relatively less gas and also that galaxies in dense environments (i.e. many galaxies per unit volume) also tend to have less gas. The imaging capability of Apertif will allow us to understand the mechanisms driving such trends. Figure 2 shows an example of the processes that influence the gas content of galaxies, based on a WSRT observation of the HI in and around a group of galaxies in a relatively dense environment. Apart from detecting the gas in the galaxies, the data also show a large cloud complex of gas in between the galaxies. This complex resulted from tidal interactions between some of the galaxies, leading to the removal of gas from these galaxies. If such interaction occur often, this may explain the lower gas content of galaxies in environments such as galaxy groups. Apertif will reveal a large number of such cases which will make it possible to obtain a complete overview of the mechanisms that control the gas content of galaxies.

In addition, the broad observing band of Apertif allows, for the first time, to detect large samples of distant (and hence younger) galaxies. This means that the evolution of gas in galaxies with redshift can be addressed. The improvement offered with Apertif will be dramatic. Currently, we know about the HI in about 40,000 galaxies, of which $99.9 \%$ are from single-dish observations of galaxies in the relatively nearby Universe out to look-back times of at most 0.5 Gyr. Hence we can only study how galaxies have evolved over such a relatively short period compared to the age of the Universe (which is about $13.7 \mathrm{Gyr}$ ). The surveys done with Apertif will result in more than one hundred thousand spatially resolved detections, most of which will be of galaxies above $z=0.05$ 
i.e. up to look-back times of 3 Gyr. Hence the evolution of the gas properties of galaxies can be studied over a much larger time span.

One of the main issues in this context is the role gas and gas accretion plays in that galaxy. The observed levels of star formation in galaxies is such that the time scale on which all gas in a galaxy should be completely consumed by star formation is typically of order of 1 Gyr, i.e. much shorter than the age of the Universe. Therefore one would expect to see a fast decrease with time of the average gas content of galaxies. In the past, the levels of star formation in galaxies were even higher, leading to even shorter depletion time scales for the gas in distant galaxies. However, this is not observed: the typical gas content of galaxies is almost constant with time, also on time scales longer than 1 Gyr. This is very puzzling and clearly something is missing in our understanding of how galaxies evolve. Apparently the decrease of the gas reservoirs in galaxies is offset by some mechanism, for example the accretion of new supplies of gas.

One possibility is that galaxies accrete gas from the inter-galactic environment. Interestingly, most of ordinary matter in the Universe is not located in galaxies, but is still 'freely floating' in the large spaces in between galaxies in the form of warm, ionised gas. Modern numerical simulations predict that, given the right conditions, part of this intragalactic gas cools and falls into galaxies, providing a channel by which galaxies can acquire fresh gas and thus keep their gas supply to higher levels. In the last decade, several deep WSRT studies have been done
NGC 4111

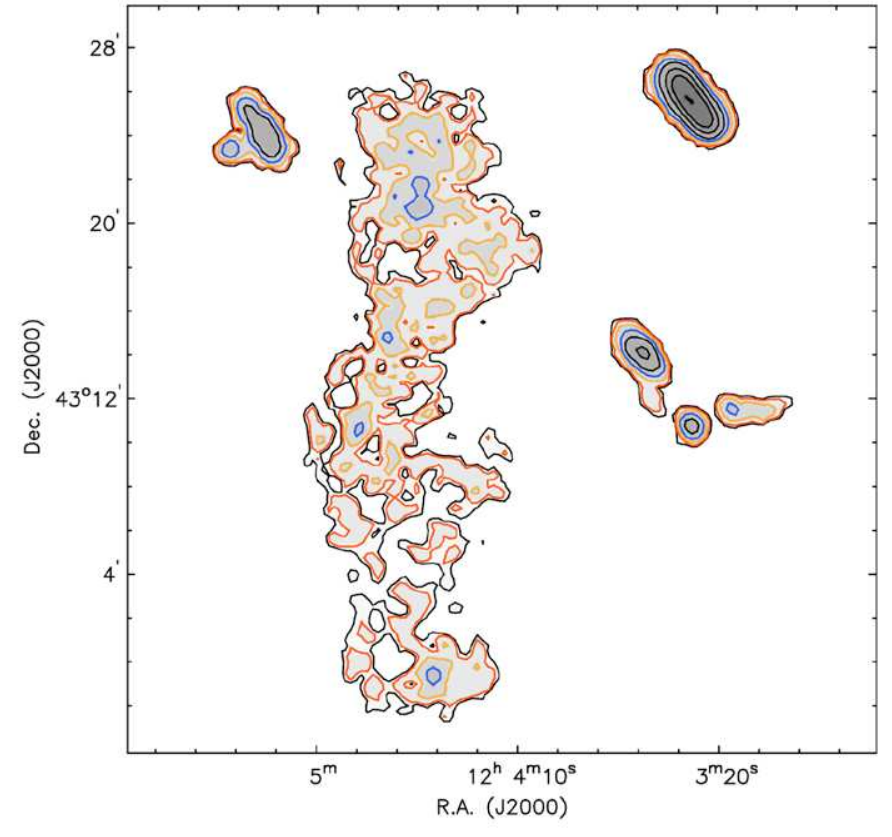

to try to detect this gas accretion and some very interesting results have been obtained. Figure 3 shows an example from a deep WSRT observation of the edge-on disk galaxy NGC 891, a galaxy very similar in size and type as our Milky Way. Given that the galaxy is seen edge-on, one can study the vertical extent of the HI. Figure 3 shows that large gas reservoirs are detected outside the galaxy. Part of this gas is connected to a gas cycle which exists in disk galaxies in the form of gas being blown out of the galaxy by supernovae connected to the star formation in the galaxy. At later times, this gas will rain back onto the galaxy. However, another part of the gas has kinematics which is not consistent with this gas cycle and is possibly gas that is being accreted from outside. Due to the large amount of observing time needed to make images like the one in Figure 3, only very few galaxies have been studied for the presence of such gas accretion and our picture of the role of accretion is very incomplete. The Apertif surveys will reveal many cases of this gas accretion and will provide crucial information on how galaxies can acquire gas and will contribute to understanding this fundamental aspect of how the gas content of galaxies evolves with time.

\section{The smallest galaxies, and smaller.}

One other key topic for Apertif is to investigate the limits of galaxy formation. The very smallest galaxies are very vulnerable objects. Their gravitational field is very weak and it does not take much to disturb such galaxies, or even to destroy them. In addition, the gas densities in these small galaxies is so low that the gas is not able to cool anymore into dense gas clouds, something which is necessary to form stars. Hence, it is expected that below a certain mass, galaxies cannot exist anymore, either because they have been destroyed by larger neighbouring galaxies, or because they were so small that no stars can have formed. It is not known what this lower limit to galaxies is. Knowing this limit is important because it gives vital information on the conditions occurring in the very early Universe when galaxies first form and which inhibit the formation of the smallest galaxies.

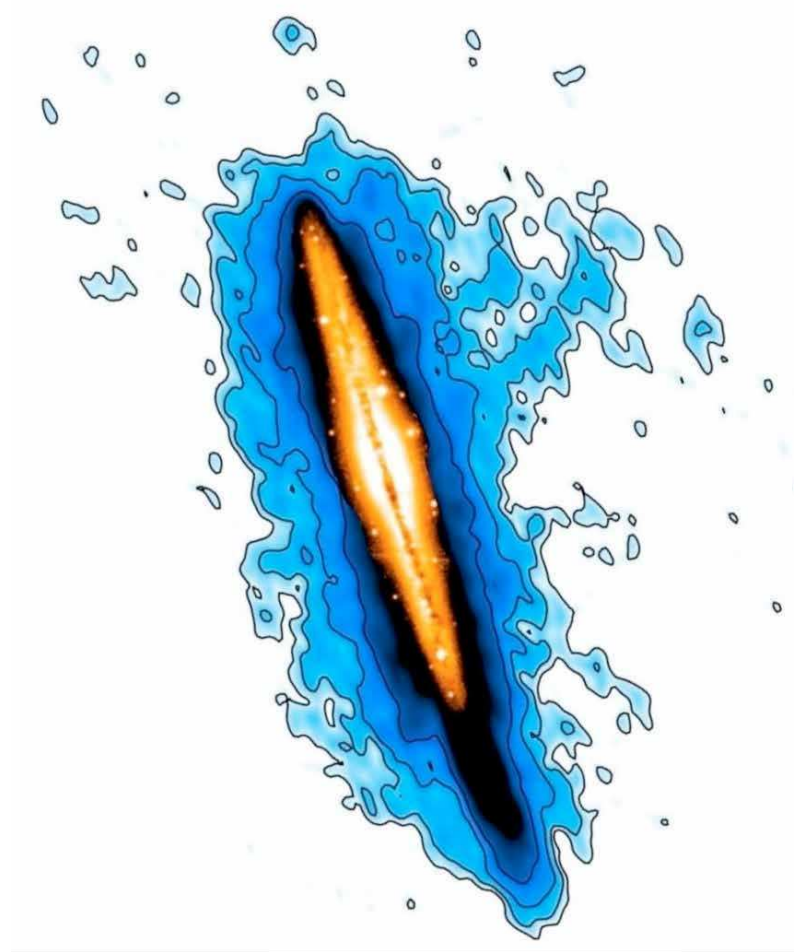

Figure 3. Image of Hit in the edge-on galaxy NCC 891 as obtained from a deep WSRT observation (Oosterloo et al. 2007). The orange depicts the stars in this galaxy, the blue the HI. The data clearly show that the HI extends well above and below the stellar disk of NGC 891 with, in particular in the NW, large streamers of gas. Detailed study of the kinematics of this gas shows that a large fraction of this extra-planar HI corresponds to HI blown out of the disk by supernovae connected to the star formation in the disk. This gas will rain down on the disk at later times. Another large part of the gas (e.g. the NW streamer) cannot be part of this gas cycle because its kinematics is not consistent with it. Possibly this 'anomalous' gas is gas that is being accreted from intergalactic space 
The smallest galaxies are expected to be very gas rich (i.e most of the ordinary matter is in the form of gas, not in stars). This is because gas may exist in the galaxy, but it is not dense enough to form stars because the gravitational potential well is not strong enough to create densities high enough for star formation. Only one or two examples of such very small, very gas rich galaxies are known. One example is shown in Figure 4 which shows Leo T as observed with the WSRT. Leo T, located about $400 \mathrm{kpc}$ from the Galaxy, has an HI mass of just above $105 \mathrm{Msol}$ and a stellar mass about 1/5-th of the HI mass, so it is gas dominated. In the optical, Leo $\mathrm{T}$ is hardly visible, but in $\mathrm{HI}$ the galaxy is very bright. Several cosmological models predict that objects like Leo $T$ should not exist because the conditions in the early Universe were such that star formation in objects of the size of Leo $\mathrm{T}$ was not possible. Yet, Leo $\mathrm{T}$ does exist indicating that our understanding of the early Universe is not complete. It is clear that we have to know much more about how many objects like Leo $\mathrm{T}$, or even smaller, exist and in which galaxy environments they are more likely to be found. In this way we obtain important constraints on what the early Universe looked like.

Because these small galaxies are small and therefore faint, they can only be detected in the very local Universe, up to distances of a few megaparsec. Hence, to survey a cosmic volume large enough to be able to detect a reasonable number of such small galaxies, the only viable strategy is to survey a large region of the sky. This will be made possible thanks to the large field of view of Apertif and it is expected that Apertif will discover a large number of systems like Leo T, providing important input for cosmological models.

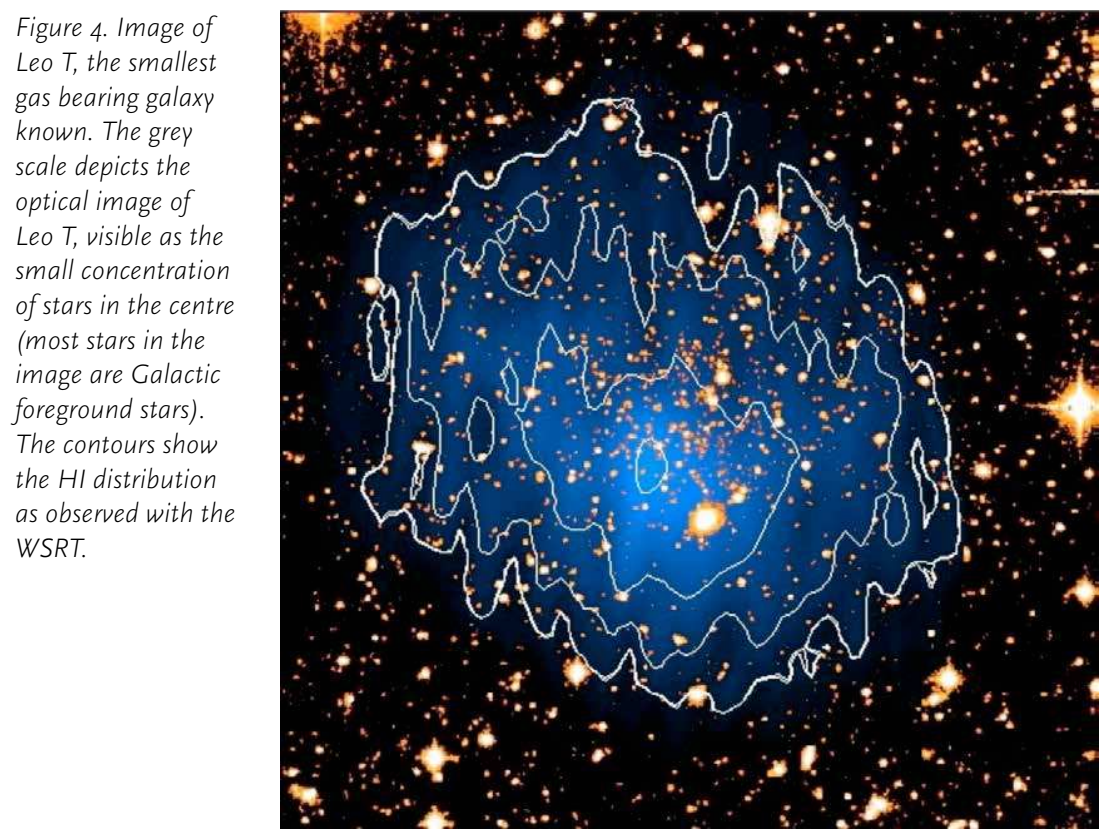

3. $\quad A G N$ and $A G N$ feedback

All large galaxies have a super-massive black hole (SMBH), typically a 100 million times more massive than the Sun, in their centre. Such a SMBH will accrete material from its immediate surroundings, making it even more massive. This is a very violent process, creating extreme physical conditions around the $\mathrm{SMBH}$. As a result, not all the accreted material falls in the SMBH, but a significant fraction is ejected away from the SMBH, often in the form of narrow jets of plasma at very high speeds (close to the speed of light). If this happens, the object is called an Active Galactic Nucleus (AGN). The plasma jets interact with the interstellar medium of the galaxy, creating massive, fast galactic outflows of gas by which galaxies can lose a significant fraction of their gas supply. This has a large impact on the ability for the galaxy to form stars because less gas means less stars can form. In particular for large galaxies, this process is thought to play an important role in their evolution.

Given the enormous amount of energy involved, one would expect that these outflows consist of hot, highly ionised gas. However, one of the surprises in the last decade, and to which the WSRT has made important contributions, has been that in many objects these fast outflows are instead mainly composed of cold (10-100 K) gas. Such cold gas outflows are detectable as very broad HI absorption profiles in the spectrum towards a bright radio continuum source (see Figure 5 for an example). It is not well understood why the outflows are predominantly cold. Given the relevance of fast gas outflows for galaxy evolution, it is important to understand this and so improve our understanding of these outflows.

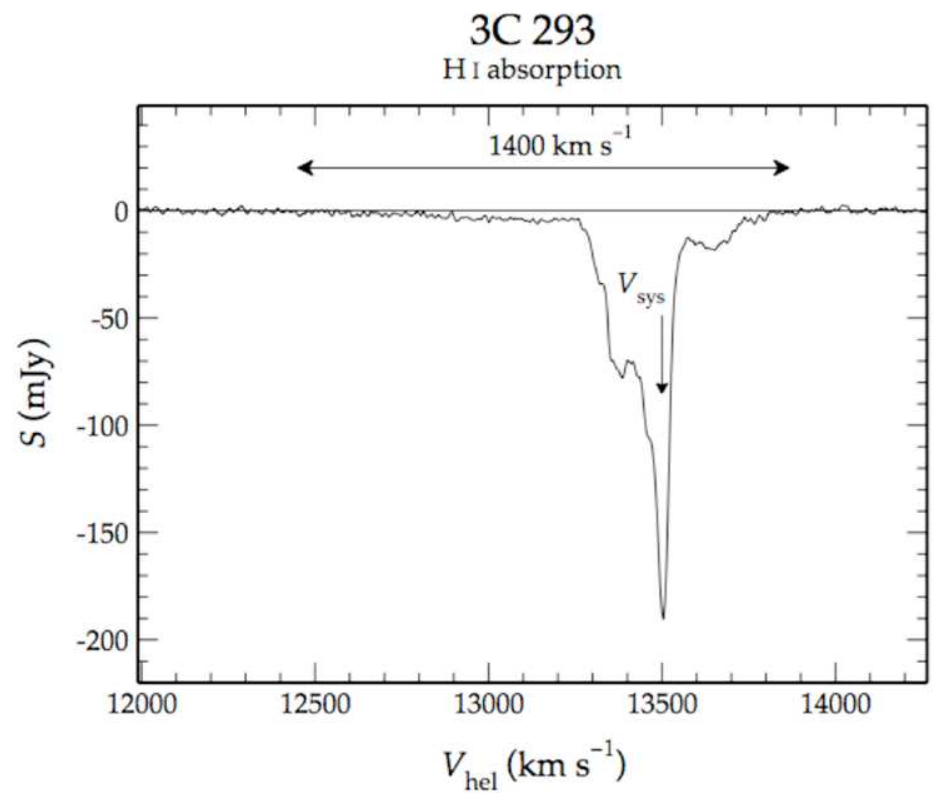

Figure 5. Detection of a fast, cold outflow in the radio galaxy 3 C293. HI absorption is detected over a very large range of velocities. The deep component is caused by the normal interstellar medium in this galaxy. The broad, shallow component toward lower velocities is due to the fast outflow of atomic hydrogen, diven by the ACN in the centre of NCC 293. 


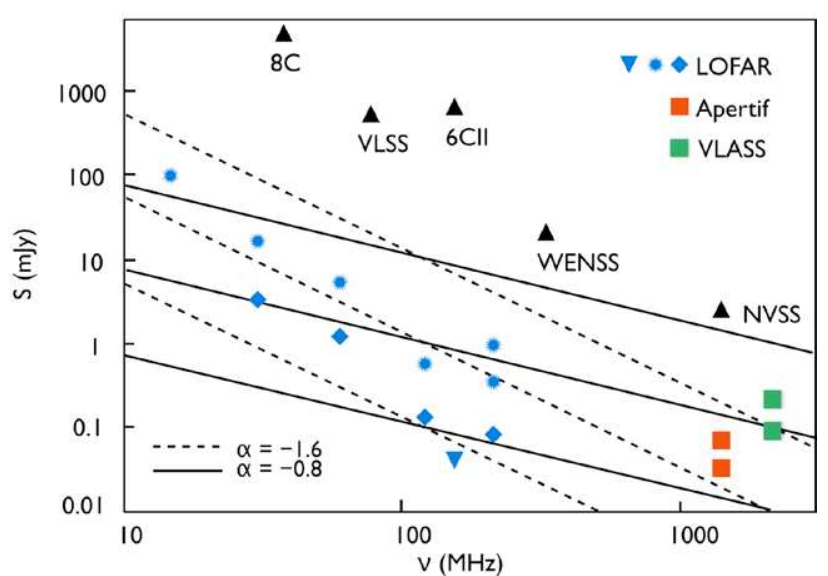

Only a few dozen cases of cold gas outflows are known. Progress has been made in understanding them, but there is a very large variety in the possible conditions around the SMBH. For example, some AGN are much more powerful than other AGN (by many orders of magnitude) and the conditions (temperature, density) of the gaseous medium around the AGN can also be very different from galaxy to galaxy. Currently available observations have not covered yet the possible parameter space, limiting a full study of these fast outflows.

Obviously, the large Apertif surveys will allow to inspect the HI spectrum towards every radio continuum AGN in the survey volume. It is expected that several hundred cold outflows will be discovered, covering a much larger parameter space than available currently. This will greatly help in making better models of the mechanisms driving these outflows. Moreover, this survey for cold outflows will be blind, i.e. not only of pre-selected subsamples of sources but of every source in the survey. This will reduce selection effects.

\section{Radio continuum and the synergy with LOFAR}

Because of the broad observing band, every Apertif observation can be used for HI science as well as for studies of the radio continuum of galaxies. This means that the Apertif HI surveys will also result in a deep, high-resolution continuum survey over the same area. The information from this continuum survey can be used to address many topics relating to galaxy evolution. For many galaxies, the radio continuum emission is caused by the star formation in these galaxies. Hence, the Apertif continuum data forms a nice complement to the HI information on the gas properties and will help address the issues discussed above.

The improvement offered by the Apertif continuum survey is very significant. For example, compared to the NVSS (the main continuum survey done with the VLA), the Apertif survey will be about a factor 50 deeper and will have a spatial resolution a factor 3 better (see Figure 6). But an important and unique strength of the Apertif continuum surveys is the synergy with the LOFAR surveys and this will remain an important advantage over any other instrumen until SKA1-Mid and SKA1-Low will become available. The relative sensitivities of LOFAR and Apertif match the spectrum of the typical radio source.This implies that most sources will be imaged by both instruments with similar signalto-noise and resolution. This, for sources detected in both surveys, flux density measurements at 60,120 and $1400 \mathrm{MHz}$ will be obtained, thus spanning more than a factor 10 in frequency range. This wide frequency coverage is extremely valuable for understanding the nature of the detected sources.The resulting radio colour-colour diagrams will be a powerful tool to spectrally discriminate between radio sources with extreme radio spectra such as diffuse emission from clusters, very distant radio galaxies and GPS/CSS sources. For nearby resolved sources it will instantly yield spectral index and spectral curvature maps, a very rich source of information to constrain many physical parameters and processes. 


\section{Exploring the time-varying Universe}

\section{Chapter 15.2 ARTS, the Apertif Radio Transient System}

Joeri van Leeuwen*

\section{Apertif on WSRT,} 2018 ompared to the pre-2017 Westerbork SRT, the factor 40 increase in fieldof-view enabled by Apertif allows for all-sky surveys with unprecedented sensitivity and speed. ARTS, the Apertif Radio Transient System, extends this wide-field Apertif system to both high time and angular resolution, enabling precision neutron-star timing, unique searches for fast transients such as pulsars and Fast Radio Bursts (FRBs), and extremely sharp imaging through VLBI. ARTS is a tied-array beamformer and a time-domain back end that turns these new receivers into high-speed and very high resolution cameras.

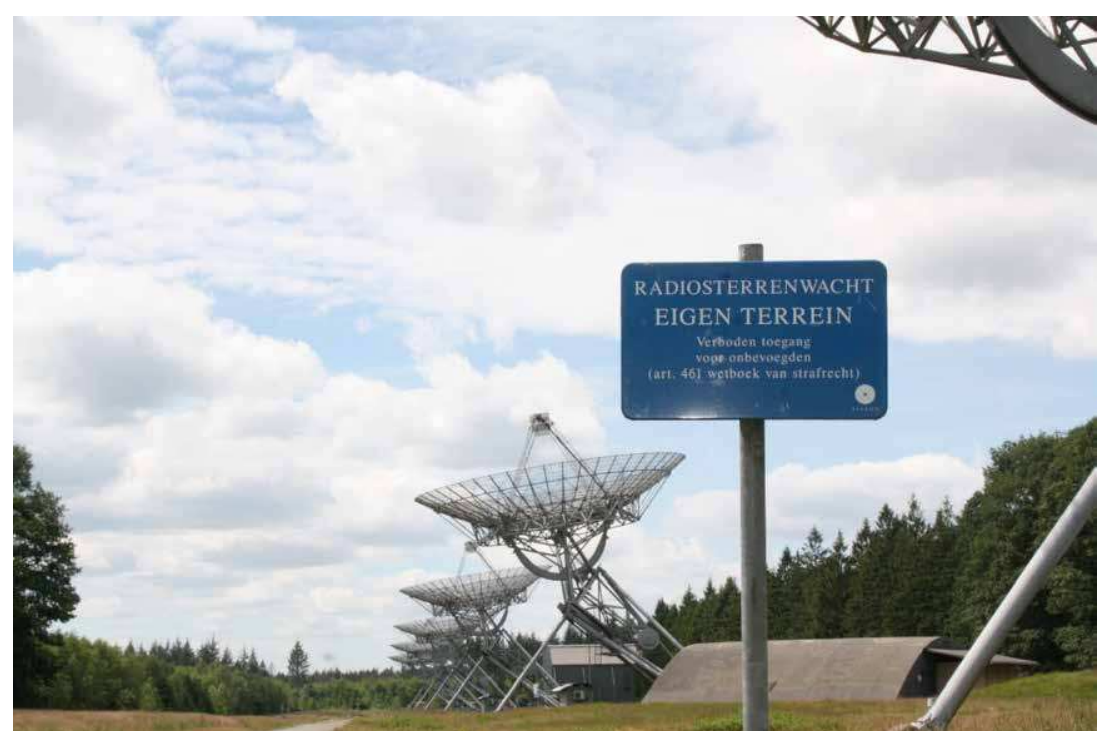

\section{Looking for new pulsars and FRBs}

Throughout the Universe, many short bright radio bursts go off. Some of these are emitted by pulsars, acting as Galactic radio lighthouses. Others originate from far outside our Galaxy, and appear to represent enormous explosions.
Such Fast Radio Bursts (FRBs) are extremely bright flashes of radio light that travel billions of light years to reach Earth. They were discovered a decade ago, but their nature continues to be largely unknown. Given the energies involved we expect that both the pulsars and the explosive bursts can provide fundamental insights into matter and gravity, which permeate our Universe. Thousands of FRBs must go off in the sky every day, but only a few dozen have been seen (Petroff et al. 2017).

To improve our understanding of pulsars and FRBs, we need find more and study these better than before. Given their unpredictability and short duration, millisecond bursts, these new discoveries can only happen with instruments that have high sensitivity, a wide field of view, and can measure fast, fleeting events.

ARTS is such an instrument: it combines the revolutionary new Apertif fron ends (the eyes), with an exceedingly powerful hybrid supercomputer (the brain), operating using new firmware and software algorithms (the thoughts). To find FRBs, ARTS continuously makes a high-speed movie of the radio sky, at 20.000 frames per second. Using that technique, we will search the entire Northern Sky to determine the origin of some of the most powerful flashes and explosions in the Universe.

\section{Successor to PuMa and PuMall}

ARTS is a hybrid FPGA-GPU machine, which serves as a cutting-edge transient survey instrument, and as a pulsar-timing and VLBI backend for all WSRT users. New firmware for the Apertif Uniboards to produces up to 468 (!) simultaneous tied-array beams -- already approaching SKA requirements, now. These can fill out the entire Apertif field of view for dedicated transient searching. Furthermore, a new Uniboard ${ }^{2}$-based second beam former that runs the same firmware will operate in parallel to any imaging that Apertif does. This truly commensal system thus provides the first ever 24/7 pulsar/FRB search machine in the world at this sensitivity and depth. Based on the system parameters and the all-sky rate, ARTS could discover one FRB per week (Maan \& van Leeuwen 2017).

After this FPGA beamforming, signals for all these applications are further processed on a large GPU cluster. This versatile back end, the successor to PuMa and PuMaII, covers the coherent dedispersion for timing, and the full-field fast-transient search. That FRB dedispersion and search on almost 500 beams needs an amount of computing power that only the fastest supercomputers in the world can produce. Thus we designed and built a GPU supercomput er, which is completely powered by image processing chips from the gaming industry. Gamers use very powerful processors for video tasks: the Graphical Processor Units (GPUs). In ARTS we now use these chips to process the high-speed time-domain streams from Apertif. The supercomputer consists of 200 GPUs, which process 4 terabits of data per second: more than the en- 

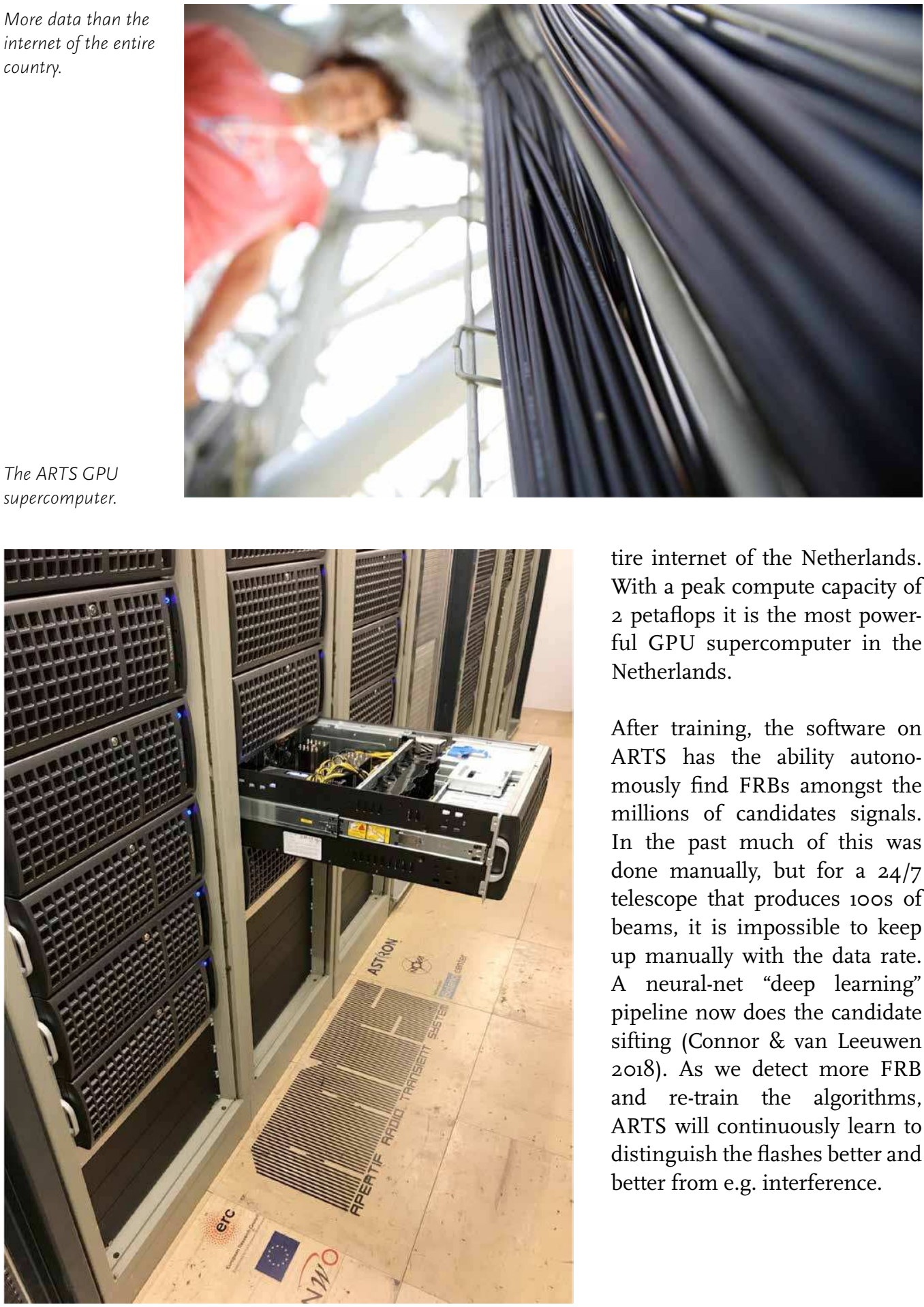

tire internet of the Netherlands. With a peak compute capacity of 2 petaflops it is the most powerful GPU supercomputer in the Netherlands.

After training, the software on ARTS has the ability autonomously find FRBs amongst the millions of candidates signals. In the past much of this was done manually, but for a $24 / 7$ telescope that produces $100 \mathrm{~s}$ of beams, it is impossible to keep up manually with the data rate. A neural-net "deep learning" pipeline now does the candidate sifting (Connor \& van Leeuwen 2018). As we detect more FRB and re-train the algorithms, ARTS will continuously learn to distinguish the flashes better and better from e.g. interference.

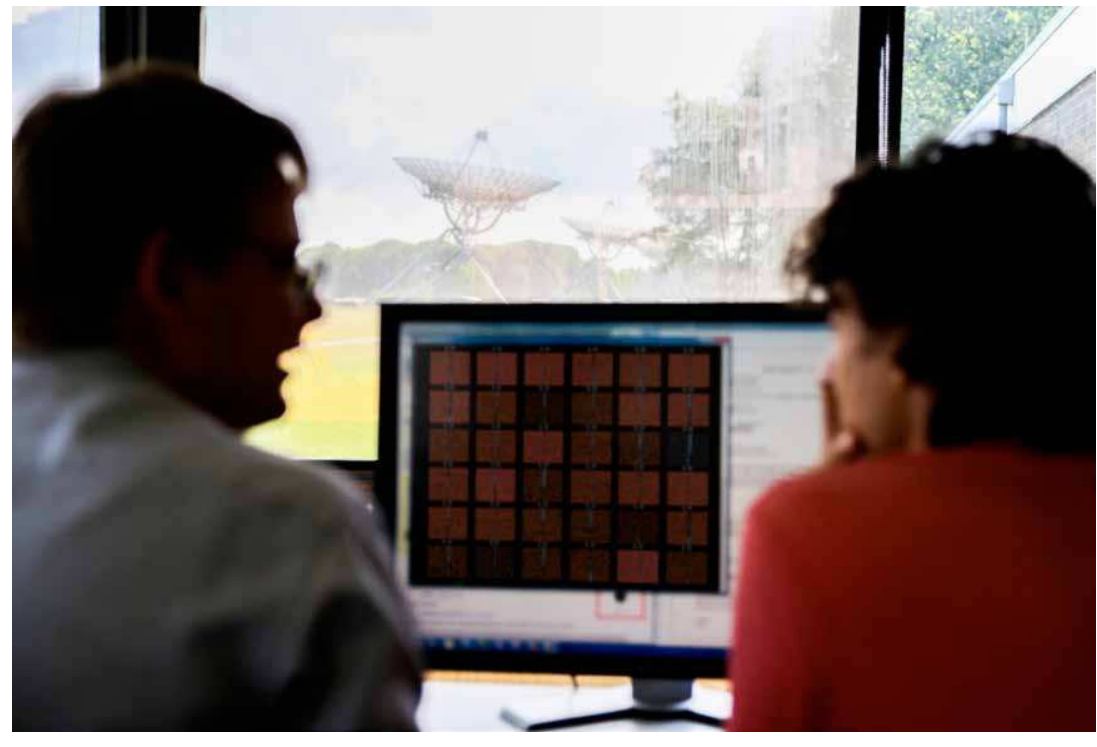

FRB Localisation

Most FRB searches are carried out using single-dish instruments, which can only provide limited localisation, preventing detailed follow-up. ARTS has the huge advantage that it employs a fully real-time interferometer, than can immediately determine the precise location of the FRBs. We know they come from other galaxies, but for one-off bursts, we have not been able to pinpoint the exact location. And so it is unknown whether e.g., all FRBs are bright bursts from neutron stars; or that exploding stars or black holes also send out these radio flashes.

In a second step of localisation, the bursts position is further refined. The FPGA-GPU supercomputer will determine where FRBs go off within a handful of seconds. Because of the dispersive delay, the same FRB will arrive at LOFAR frequencies tens of seconds later. By triggering the LOFAR Transient Buffer Boards at the -- now known -- correct direction, arrival time and dispersion measure, these FRBs can be even better localised. We thus take two of the best radio telescopes in the world -- and combine them to produce a burst-finding machine that is far more powerful than the sum of its parts. 


\section{Apertif; the next stage}

\section{Chapter 15.3 From Multi-frequency to Widefield; the System}

\author{
Wim van Cappellen, Gert Kruithof*
}

$\mathrm{T}$

ability to survey the radio sky to high sensitivity and at high resolution provides a powerful probe of a number of key questions in modern astronomy. In this fiftieth year of the WSRT, ASTRON and partners are finalising the major upgrade of 12 dishes of the Westerbork Array, termed 'Apertif', to become one of only two arrays equipped with powerful phased array receiver systems for that purpose. In the first part of this chapter, Tom Oosterloo sets out the exciting science case for developing such a powerful GHz-range wide field survey instrument. In the second chapter part, Joeri van Leeuwen details the instrumental expansion with ARTS to be able to observe the time-varying universe in combination with Apertif. In this third contribution, the scope and design of the combined Apertif-ARTS system are presented which will ensure that the WSRT performs as a world-leading instrument for many years to come.

\section{The APERTIF-ARTS system}

The WSRT upgrade from multi-frequency front end (MFFEs) receivers to one utilising Phased Array Feeds delivers a telescope capable of multi-beam observing such that a whole new scope of astronomical research can be realised. In this upgrade, the WSRT dishes are reused with only minor modifications, whereas the remainder of the WSRT systems are replaced with innovative technologies.

The Apertif-ARTS system is a unique telescope array in the northern hemisphere. Together with ASKAP (the Australian SKA Pathfinder) in Australia, it provides the possibility of surveying the whole sky. As an imaging telescope, the system operates with a multi-pixel aperture synthesis array on 12 dishes in dual polarization, at frequencies $1130-1750 \mathrm{MHz}$ with a maximum baseline length of about $2.5 \mathrm{~km}$. The ARTS aspect of this system is dedicated to tranthe system observes the sky over a remarkably large field of view (8 square degrees, about 3ox larger than with the MFFE receivers). This system also has the ability to process $300 \mathrm{MHz}$ instantaneous bandwidth - another factor of two compared to the MFFE system.

The Apertif-ARTS system has four main parts: phased array feeds in the focus of each dish, a cabin below each dish where the signals are conditioned, digitized and beam formed, a correlator in the central building to combine the signals of all dishes in parallel, and a tied-array backend. An overview of the system is shown in Figure 1.

The system components from the feeds to the correlator/beam former are com mon to both the APERTIF (imaging) and ARTS (time domain) aspects. It is only post-correlator/beam former that the two systems differ.

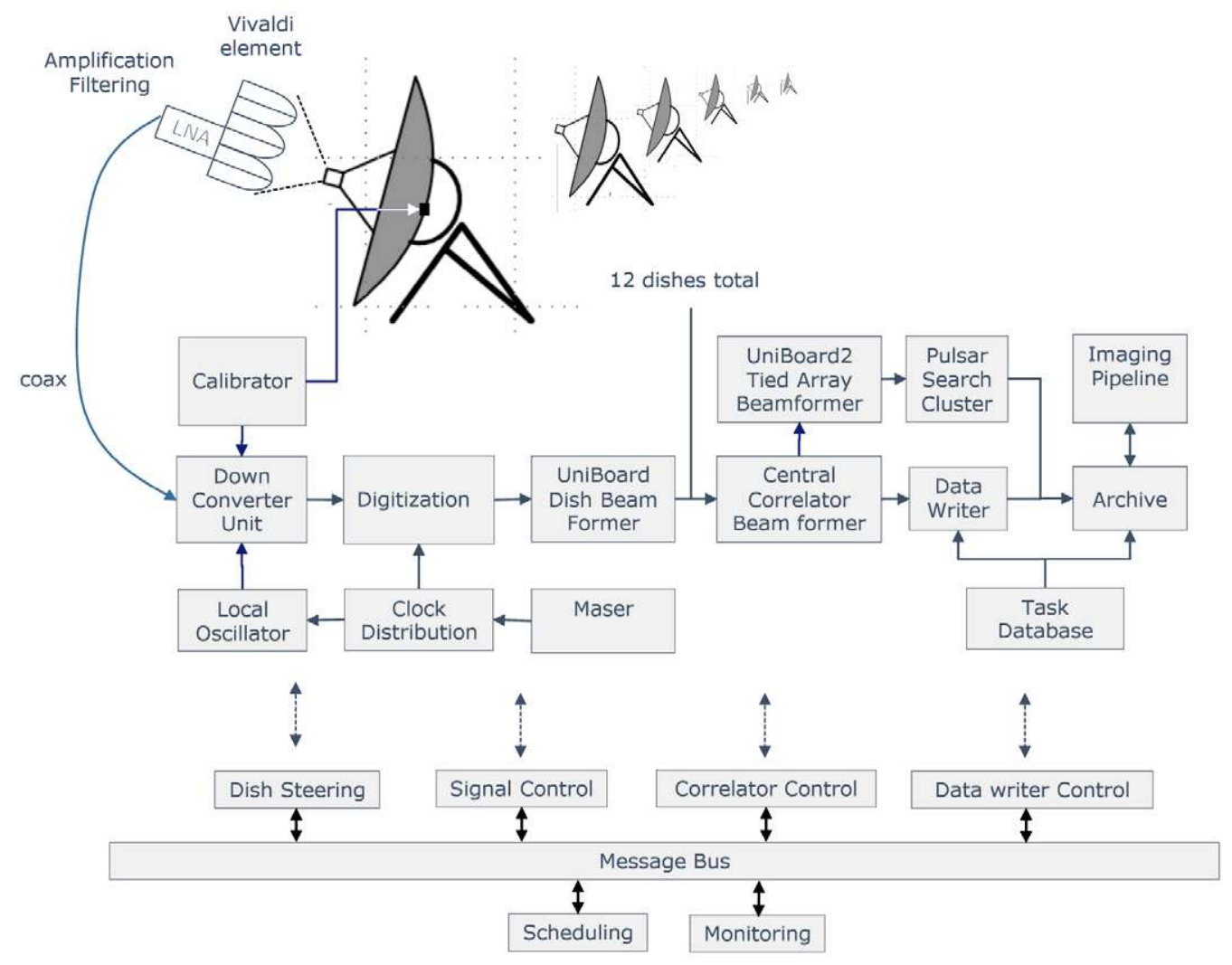

During observations radio signals from the sky are detected by the phased array Figure 1 Overview of feeds in the focus of the dishes. These uncooled arrays are each made up of 121 the APERTIF ARTS Vivaldi antenna elements, including the Low Noise Amplifiers, arranged in two system orthogonal polarisations (Figure 2). A summary of the Apertif-ARTS system parameters is shown in Table 1. 
Figure 2 The phased array feed of the Apertif system as mounted in the prime focus box of

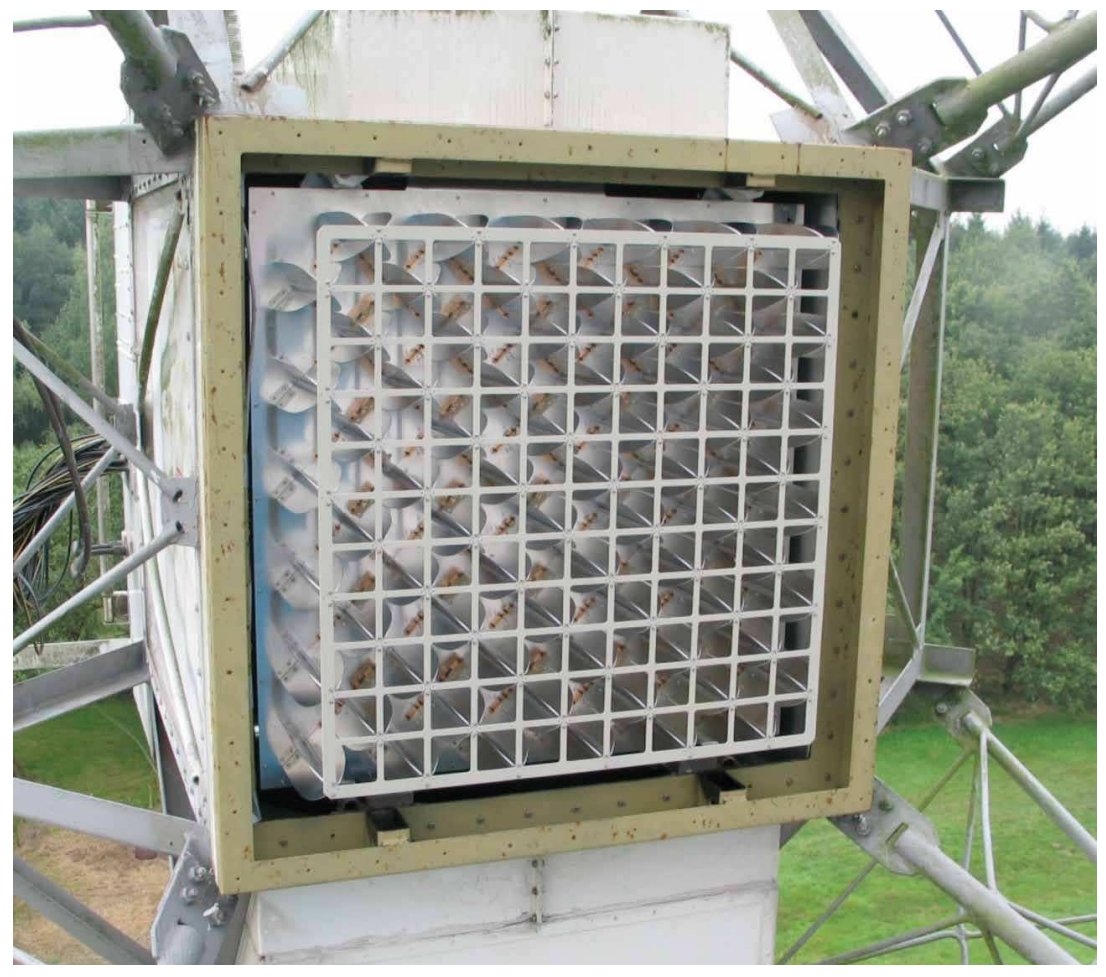

Table 1: Overall System parameters

\begin{tabular}{l|l}
\hline Parameter & Value \\
\hline Field of View & 8 square degrees \\
\hline Number of beams & 40 \\
\hline Angular resolution at $1.4 \mathrm{GHz}$ & $13 \times 13 / \mathrm{sin}(\delta)$ arcsec2 (EW by NS) \\
\hline System temperature & 70 Kelvin \\
\hline Aperture efficiency & 0.75 \\
\hline Frequency range & $1130-1750 \mathrm{MHz}$ \\
\hline Instantaneous bandwidth & $300 \mathrm{MHz}$ \\
\hline $\begin{array}{l}\text { Number of channels } \\
\text { (=\#subbands x \#channels/sub.) }\end{array}$ & $384 * 64=24576$ \\
\hline Noise spectral line (12 hr) & $0.5 \mathrm{mly} /$ beam over $16 \mathrm{~km} / \mathrm{s}$ \\
\hline Noise continuum (12 hr) & $10 \mu / \mathrm{y} /$ beam over $300 \mathrm{MHz}$ \\
\hline $\begin{array}{l}\text { Noise uniformity over Field-of-View } \\
\text { Primary beam size of one beam }\end{array}$ & $10-25 \%$ \\
\hline $\begin{array}{l}\text { Survey speed increase compared to } \\
\text { WSRT-MFFE system }\end{array}$ & 33 arcmin \\
\hline
\end{tabular}

After filtering and amplification, the radio frequency signals are transported over coaxial cables to the telescope cabins. Here the signals are conditioned, digitized and beam formed. After conversion to an intermediate frequency, the signals are digitised by 8 -bit analogue to digital converters at a sampling clock rate of $800 \mathrm{MHz}$. The raw data rate per phased array feed for two polarizations is $800 \mathrm{Gbps}$, i.e. then effectively $300 \mathrm{MHz}$ is digitized into $781 \mathrm{kHz}$ wide sub bands. Each of these bands is processed independently.

Figure 3 shows one Apertif dish beam former capable of processing a single polarization of one phased array feed. It shows a backplane board with eight digitizer boards plugged into the left side and two of the four UniBoards used (see below and also Chapter 10) mounted at the right side. The dish beam former combines the responses of all elements into 40 compound beams, reducing the data rate by a factor of almost 3 . Each dish uses an online calibration system to stabilise the compound beam patterns. A switched noise source in the apex of each dish is used to monitor and compensate temporal gain and phase variations of the receiver chains. Each dish beam former sends its output over optical fibre to the central signal processing unit which then either correlates the signal for imaging observations (APERTIF) or beam forms the signal for time domain observations (ARTS).

The combined data rate into the central correlator/beam former for all 12 dishes is about 3.5Tbps: this makes it one of the most powerful signal processing systems used in Radio Astronomy today. During imaging observations, we can

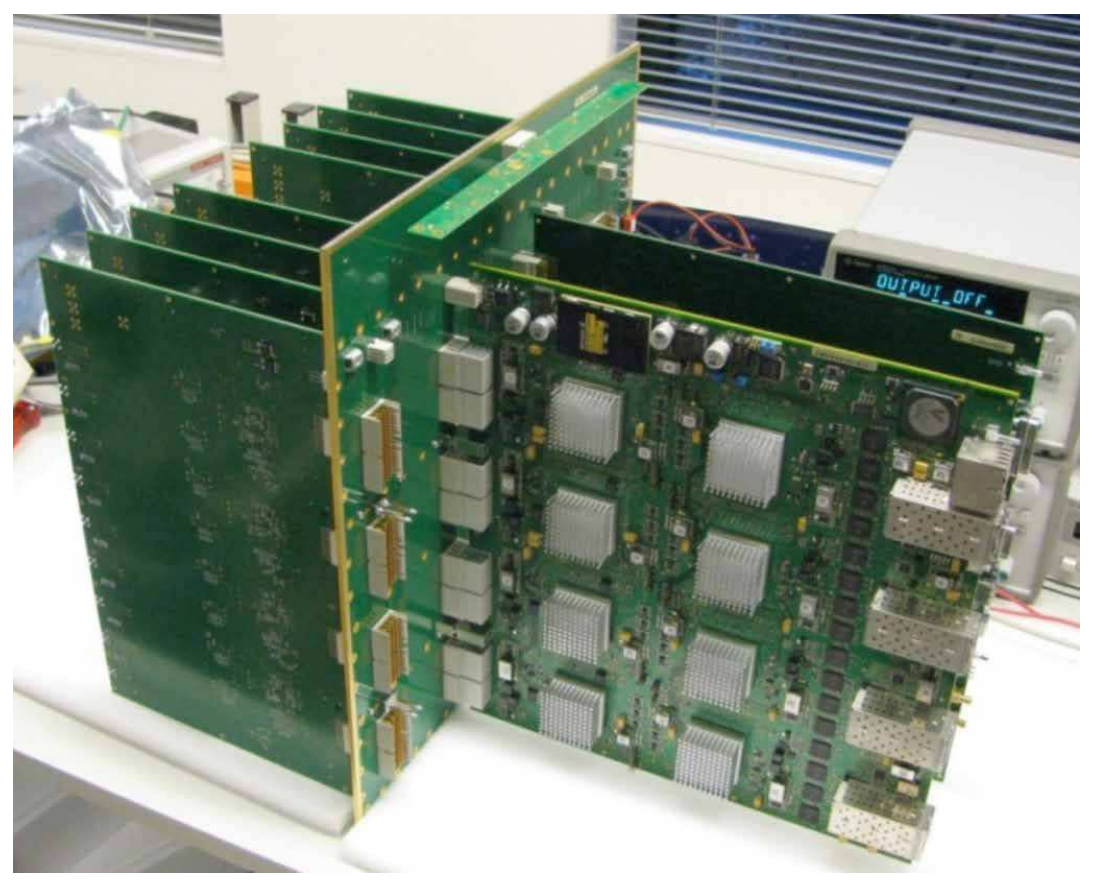

Figure 3 The Analog to Digital conversion on the backplane of the Dish Beam Former Boards (housing removed) 
also do commensal observations of radio transients via the dedicated tied array beam forming unit, thus maximising the scientific output of each observation. ARTS can create up to 12 tied array beams to fill a single compound beam.

The system uses both first and second-generation versions of the powerful "UniBoard" digital signal processing systems. These systems have been developed by ASTRON and partners in the EC-FP7 Radionet3 program. These boards have been specially designed to handle the extreme data streams in real time. The dish beam formers and the central correlator/beam former use the first generation UniBoard. Each dish has 8 UniBoards (4 per polarisation), and the central signal processing unit has 16 UniBoards. Each UniBoard has 8 FPGA's (Field Programmable Gate Arrays), storage memory and about 10.000 connections in 14 Printed Circuit Board layers between the components. Interestingly, this single board has about the same number of connections between the components as shown on the backplane of the DLB (see Chapter 10). In correlator mode, the complex sub bands of each telescope beam are Fourier transformed into 64 channels of about $12 \mathrm{kHz}$ width, cross multiplied and integrated.

The tied-array backend for transient surveys, pulsar timing and VLBI uses UniBoard2. This second generation UniBoard is the first of its type to use the Arriaio $20 \mathrm{~nm}$ chip and also the first such system that can achieve a processing rate exceeding 1 Tbps.

A Monitoring and Control framework is used to schedule each observation, steer all the components in the signal chain and monitor the entire system during observations. The framework is

\section{An overview of the Apertif Phased Array Feed principles cert Kruthof, Carole acckson}

The key topics of an international workshop in Sydney, Australia 2017: www.pafworkshop.org ) emphasised the technologies, science and applications of so-called Phased Array Feeds (PAFs). Whilst the focus was on Radio Astronomy as the originating discipline, the use of PAFs in other applications were noted e.g. in the next generation 5 telecom infrastructures. This, and other similar reviews demonstrate the potential of the PAF technology. Having been steadily developed over the last two decades, we are at the time when PAFs are mature and installed as 'live' systems.

Recognising the potential of aperure arrays in general for either or both of SKA and LOFAR, ASTRON commenced research in the underlying concepts in the mid-nineties. This provided the foundation for ASTRON to drive the concept to maturity with the Apertif system for the Westerbork Synthesis Radio Telescope
Whilst other parts of this contribution discuss the details of th innovations demanded by the PAFs, we take this opportunity to summarise how these systems work in principle:

We should first consider the case of a single dish telescope: A single pixel horn feed mounted at prime focus of a parabolic dish is sensitive to the incoming radiation (a plane wave) from an object aligned with the central axis of the dish. In fact only the incoming waves on the cenred in the feed area of the focal plane. Incoming waves at other angles are not detected.

To detect off axis signals requires a larger area receiver in the focal plane region. The challenge has been to design a receiver of many adjacent antennas, spaced less than half one wavelength apart.

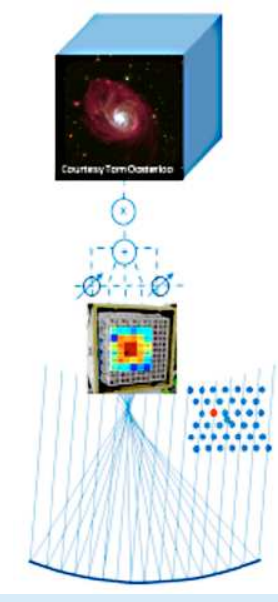

5. The end result after calibration and imoging is an

\section{The signals from 12 dishes are then correlated}

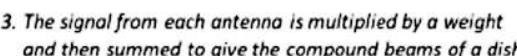
2. Multiple Vivoldi ontennas ore required to somple the signal in the focal plane. 1. Plane woves ot an angle to the central plane (shown here for one direction). The compound beam pottern is chosen to ollow easy mosaicking

In this type of receiver, each antenna collects a part of the signal at the focal plane. By applying weights to the signals from each antenna before summation, we can reconstruct the wave across a wide area of the focal plane (and arrives at angle In fact this type of receiver allows for fact, this type of receiver allows the signals received resulting in a high efficiency array feed with small smooth frequency response. The phased array feed in the ASTRON Apertif system is one manifestation of a focal plane feed with an array of 121 Vivaldi antennas in each receiver. This receiver collects signals from a circular area of 8 square degrees on the sky. This area is distributed across 40 beams arranged in a hexagonal pattern. Each beam is formed (in our terminology we call this 'beamforming') by adding the signals of a subset of antennas, with each signal assigned a very specific weight. Obviously, this demands intense, high-data-rate signal processing techniques to make it all work part of the Apertif system.

Secondly, what does it mean for Apertif as a survey (and more!) instrument? Sometimes termed a radio 'camera', the array feed is termed as 'dense' - it is able to form tens of optimised ('shaped') and overlapping compound beams on the sky electronically As this type of phased array feed is uncooled, it feed is uncooled it The nos tempient temperature. The noise temperature of each beam is higher than the equivalent single pixel (e.g. horn) cryogenically cooled feed. However, Apertif has 40 instantaneous beams that more than compensate for this. Combined with the increased efficiency (i.e. less loss of signal) a net survey speed increase of the WSRT of 15 times results (see Tabler). 
based on a Service Oriented Architecture allowing a flexible, loose coupling between all the components. Each hardware component uses drivers that connect as a service to the message bus. A workflow orchestrates the components in the signal chain. Each component provides information on the message bus on its status. These messages are collected in the successor of the TMS database (see Chapter 10), called Artamis to monitor the system health, and quality of the observations.

During imaging observations, the data flow from the correlator to the data writer, creating a measurement set in the Apertif Long Term Archive (ALTA): ALTA comprises 1.5 petabyte of disk storage used to store the data for further processing. The measurement sets can be processed off-line with the "Apercal" pipeline to produce higher-level data products such as data cubes and catalogues. In beam forming mode, the data flows to the ARTS GPU cluster. This cluster uses machine learning for automatic detection of transient candidates. These are analysed further in pipelines. The same backend is also used for pulsar search and timing.

All resultant data is published to the ALTA long-term archive which is designed to scale up to 20 petabyte of storage. This data is publicly available, forming a legacy dataset ready for use across a wide range of astronomical research. 